\title{
Title
}

\section{- Highly diverse Asgard archaea participate in organic matter degradation in coastal} sediments

- Asgard archaea are capable of organic matter utilization

\section{Authors}

Mingwei Cai1 ${ }^{1,2 \dagger}$, Yang $\mathrm{Liu}^{1 \dagger}$, Xiuran $\mathrm{Yin}^{3,4}$, Zhichao Zhou ${ }^{1,5}$, Michael W. Friedrich ${ }^{3,4}$, Tim Richter-Heitmann ${ }^{3}$, Rolf Nimzyk ${ }^{6}$, Ajinkya Kulkarni ${ }^{3}$, Xiaowen Wang ${ }^{1,2}$, Wenjin Li $^{1}$, Jie Pan $^{1}$, Yuchun Yang ${ }^{5}$ Ji-Dong $\mathrm{Gu}^{5}$ and Meng $\mathrm{Li}^{1 *}$

\section{Affiliations}

${ }^{1}$ Institute for Advanced Study, Shenzhen University, Shenzhen, China.

${ }^{2}$ Key Laboratory of Optoelectronic Devices and Systems of Ministry of Education and Guangdong Province, College of Optoelectronic Engineering, Shenzhen University, Shenzhen, China.

${ }^{3}$ Microbial Ecophysiology Group, Faculty of Biology/Chemistry, University of Bremen, Bremen, Germany.

${ }^{4}$ MARUM, Center for Marine Environmental Sciences, University of Bremen, Bremen, Germany.

${ }^{5}$ Laboratory of Environmental Microbiology and Toxicology, School of Biological Sciences, The University of Hong Kong, Pokfulam Road, Hong Kong SAR, China. ${ }^{6}$ Department of Microbe-Plant Interactions, Faculty of Biology/Chemistry, University of Bremen, Bremen, Germany 
*CORRESPONDENT: Meng Li, Room 360, Administration Building, Institute for

Advanced Study, Shenzhen University, Shenzhen, China; E-mail: limeng848@szu.edu.cn;

Tel: $+86-755-26979250$.

TThese authors contributed equally to this work.

\section{ABSTRACT}

Asgard is an archaeal superphylum that might hold the key to understand the origin of eukaryotes, but its diversity and ecological roles remain poorly understood. Here, we reconstructed 15 metagenomic-assembled genomes (MAGs) from coastal sediments covering most known Asgard archaea and a novel group, which is proposed as a new Asgard phylum named as the "Gerdarchaeota". Genomic analyses predict that Gerdarchaeota are facultative anaerobes in utilizing both organic and inorganic carbon. Unlike their closest relatives Heimdallarchaeota, Gerdarchaeota have genes encoding for cellulase and enzymes involving in the tetrahydromethanopterin-based Wood-Ljungdahl pathway. Transcriptomic evidence showed that all known Asgard archaea are capable of degrading organic matter, including peptides, amino acids and fatty acids, in different ecological niches in sediments. Overall, this study broadens the diversity of the mysterious Asgard archaea and provides evidence for their ecological roles in coastal sediments. 


\section{MAIN TEXT}

\section{Introduction}

Asgard archaea, proposed as a new archaeal superphylum, are currently composed of five phyla, i.e., Lokiarchaeota ${ }^{1}$, Thorarchaeota ${ }^{2}$, Odinarchaeota $^{3}$, Heimdallarchaeota ${ }^{3}$, and Helarchaeota $^{4}$, some of which encompasses lineages formerly named Marine Benthic Group B $(\mathrm{MBG}-\mathrm{B})^{5}$, Deep-Sea Archaeal Group (DSAG) ${ }^{6}$, Ancient Archaeal Group (AAG) ${ }^{7}$, and Marine Hydrothermal Vent Group $(\mathrm{MHVG})^{7,8}$. Since Asgard archaea contain abundant eukaryotic signature proteins (ESPs) and form a monophyletic group with eukaryotes in the phylogenetic tree inferred from 55 concatenated archaeo-eukaryotic ribosomal proteins, they are regarded as the closest relatives of Eukarya and have attracted increasing research interests ${ }^{1,3,9}$.

Metabolic potentials of several Asgard phyla have been predicted based upon their genomic inventories: hydrogen dependency ${ }^{10}$ in Lokiarchaeota (which were originally described in deepsea sediments), mixotrophy ${ }^{11}$ (i.e., using both inorganic and organic carbon for growth) and acetogenesis $^{2}$ by Thorarchaeota from estuary sediments, metabolization of halogenated organic compounds $^{12}$ by Loki- and Thorarchaeota, phototrophy ${ }^{13,14}$ in Heimdallarchaeota from coastal sediment and hydrothermal vent samples, anaerobic hydrocarbon oxidation in hydrothermal deepsea sediment by Helarchaeota ${ }^{4}$, and nitrogen and sulfur cycling ${ }^{15}$ in all Asgard archaeal phyla. Metatranscriptomics revealed a few transcripts encoding NiFe hydrogeneases from deep-sea Lokiarchaeota $^{16}$. Since no comprehensive information about the in situ activity of these Asgard archaea has been compiled so far, our understanding of these phylogenetically and evolutionally important archaea is based on prediction, and thus, severely limited.

Coastal environments, e.g., mangroves, salt marshes and seagrass beds, are known sinks of blue carbon ${ }^{17}$. Although these vegetated coastal ecosystems make up less than $0.5 \%$ of the seabed, they hold $\sim 50 \%$ of organic carbon of the surface marine sediments globally ${ }^{17-19}$. Here, we reconstructed 15 Asgard metagenomic-assembled genomes (MAGs) from diverse coastal 
68 sediments and analysed them together with transcriptomes from mangrove sediments to clarify

69 the ecological roles of the different Asgard clades in these important environments. Based on

70 phylogenetic analysis of this dataset, we propose a novel Asgard phylum, the "Gerdarchaeota".

71 Additionally, we recruited all publicly available $16 \mathrm{~S}$ rRNA sequences to see whether or not

72 distinct Asgard lineages show distinct habitat preferences. Our findings substantially extend our

73 knowledge of the lifestyles of these mysterious archaea in coastal sediments and their ecological

74 roles on carbon cycling.

75

\section{Results and Discussion}

\section{Mining Asgard archaea genomes lead to the proposal of a new Asgard phylum}

Sediments from several coastal sites (mangrove, mudflat and seagrass bed) were collected for deep metagenomic and metatranscriptomic sequencing (totally $2.3 \mathrm{Tbp}$, table $\mathrm{S} 1$ ). By combining individual samples from the same site but from different depth layers for assembly and binning, we recovered 15 Asgard MAGs with completeness of $>80 \%$ through hybrid binning strategies $\left(\right.$ MetaBAT $^{20}$ in combination with Das Tool ${ }^{21}$ ) and manual bin refinement. Based on a phylogenetic analysis with a concatenated set of 122 archaeal-specific marker genes, we found that eight of the MAGs belong to known Asgard lineages, covering almost all phyla (except Odinarchaeota), i.e., Helarchaeota (1 MAG), Lokiarchaeota (2), Thorarchaeota (3), Heimdallarchaeota-AAG (1), and Heimdallarchaeota-MHVG (1) (Fig. 1A and table S2). The remaining 7 MAGs clustered with an unclassified MAG (B18_G1) and formed a monophyletic group in phylogenetic tree of concatenated 122 archaeal marker genes (Fig. 1A).

The amino acid identity (AAI, $43 \%$ to $50 \%$ compared to other Asgard archaea), pan-genome analysis, and non-metric multidimensional scaling (NMDS) ordination agreed on assigning phylum level identity to this new clade, (figs. S1, S2 and S3). Likewise, phylogenetic analysis of a 16S rRNA (1152 bp) gene found in of those seven MAGs (YT_re_metabat2_2.057) showed that 
93 it formed a new monophyletic branch (Fig. 1B) with an identity below 74\% to 16S rRNA genes

94 of other Asgard archaeal phyla (table S3). Thus, we propose this lineage as a new phylum named

95 Gerdarchaeota, after Gerd, the Norse goddess of fertile soil, because these Asgard archaea

96 genomes were obtained from organic-rich coastal environments ${ }^{22}$, such as mangrove, mudflat and 97 seagrass (table $\mathrm{S} 1)$.

The presence of eukaryotic signature proteins (ESPs) is a characteristic feature of Asgard (Fig. 1C and table S4), and we consistently found that their phylogenies support the discrete position of Gerdarchaeota in the Asgard lineage (fig. S4). Homologs encoding eukaryotic-type topoisomerase IB and fused RNA polymerase subunit A were identified in Gerdarchaeota, while neither genes for eukarya-specific DNA polymerase epsilon and DNA-directed RNA polymerase subunit $\mathrm{G}$ were detected (Fig. 1C, and table S5). Gerdarchaeotal topoisomerase IB is clustered into a sister group of Thorarchaeota and the two Asgard sequences together were monophyletic with high supporting values (fig. S4A). The fused RNA polymerase A genes of Gerdarchaeota are transcriptionally expressed (table S5) and clustered into a sister group of Heimdallarchaeota, and those of Helarchaeota branch with Loki- and Thorarchaeota (fig. S4B). In both cases, the eukaryotic genes do not cluster with the Asgard homologs. Besides, Gerdarchaeota also comprise expressed homologues of ribophorin I and STT3 subunit and lack OST3/OST6 homologues in most genomes except YT_bin5.010. The phylogenetic tree of ribophorin I showed that Gerdarchaeota is monophyletic and branches with Heimdallarchaeota, and the eukaryotes branch within the cluster of Thor-, Hel- and Lokiarchaeota (fig. S4C). In addition to the reported ESPs, we identified DAD/OST2 homologues within Gerdarchaeotal MAGs, which is a component of the $\mathrm{N}$-ologosaccharyl transferase for the $\mathrm{N}$-linked glycosylation ${ }^{23}$.

\section{Metabolic potential of the new phylum Gerdarchaeota}


117 Gerdarchaeota harbour the gene set for oxidative phosphorylation, including V/A-type ATPs,

118 succinate dehydrogenase, NADH-quinone oxidoreductase, the key enzyme cytochrome $c$ oxidase

119 (subunits I, II, and III) (Fig. 2, fig. S5, and tables S6 and S7), and enzymes for the non-typical

120 cytochrome $b c l$ complex (i.e., SoxL) ${ }^{24}$ but lacking the genes for other respiratory complex III

121 (e.g.,SoxN and CbsA), suggesting that Gerdarchaeota most likely perform aerobic respiration.

122 Gerdarchaeotal cytochrome $c$ oxidases are phylogenetically separated into two lineages (fig. S6),

one of which clusters closely with the facultative anaerobic Crenarchaeotal Acidianus brierleyi ${ }^{25}$,

$124 \quad 26$ and the other one groups with the bacterial Synechocystis sp., which is capable of aerobic

respiration in the dark ${ }^{27}$. Meanwhile, Gerdarchaeotal MAGs harbour genes encoding

126 heliorhodopsins (fig. S7), which might sense light in the top layers of sediment ${ }^{13}, 14$. A complete

127 tricarboxylic acid cycle (e.g., citrate synthase and malate dehydrogenase, Fig. 2) further supports

128 aerobic respiration as important dissimilatory pathway. Besides, Gerdarchaeota are equipped with

129 enzymes for the conversion of $\mathrm{AsO}_{4}{ }^{3-}$ to $\left(\mathrm{CH}_{3}\right)_{n}-\mathrm{As}$, thus they could remove the toxic $\mathrm{As}(\mathrm{V})$ and

130 As(III), which can disrupt oxidative phosphorylation and inhibit respiratory enzymes ${ }^{28}$.

131 Within Gerdarchaeota MAGs, complete gene sets for acetogenesis pathways (e.g., acetyl-

132 CoA hydrolase) are present (Fig. 2), implying the ability of these archaea to perform fermentation

133 in the absence of oxygen. We further identified all subunits of [NiFe]-hydrogenase heterodisulfide

134 reductase $h d r A B C$. Since this community does not contain the key enzymes $m c r A B C$ for

135 methanogenesis, $h d r A B C$ might function to accept electrons and reduce oxidized ferredoxin

136 instead of a heterodisulfide anaerobically ${ }^{29-32}$. Additionally, we identified homologues for ferric

137 reductase but their capability to use Fe(III) as electron acceptors remained open. Notably, the

138 canonical nitrate reductase (previously identified in Heimdallarchaeota ${ }^{32}$ ) was not detected in

139 Gerdarchaeota MAGs.

140 Gerdarchaeota appear to use diverse organic compounds (e.g., formaldehyde, amino acid,

141 peptide, lipid and ethanol) as electron donors (Fig. 2). For genes encoding peptidase, serine 
142 peptidases dominated ( $44.1 \%$ of total peptidase, fig. S8A). We also identified abundant genes

143 for cellulose degradation (e.g., AA3 and GT2, fig. S8B and table S8), which may be further

144 degraded through the Embden-Meyerhof-Parnas (EMP) pathway. Different from the facultative

145 anaerobic relatives Heimdallarchaeota, Gerdarchaeota contain the complete genes for

146 tetrahydromethanopterin Wood-Ljungdahl (THMPT_WL) pathway and the key enzyme acetyl-

147 CoA decarbonylase/synthase (Fig. 2). The presence of the genes for groups $3 b$ and $3 c[\mathrm{NiFe}]-$

148 hydrogenases (fig. S9) implies that these archaea may grow lithoautotrophically using $\mathrm{H}_{2}$ as

149 electron donor as also reported for Lokiarchaeota ${ }^{10}$. Meanwhile, we identified other $\mathrm{CO}_{2}$

150 assimilation pathways in Gerdarchaeota MAGs. For example, Gerdarchaeota are equipped to fix

151 carbon via the complete reductive citric acid cycle (e.g., 2-oxoglutarate oxidoreductase and

152 isocitrate/isopropylmalate dehydrogenase) and Calvin-Benson-Bassham (CBB) cycle, and the

153 pyruvate dehydrogenase, which is responsible for the generation of pyruvate from acetyl-CoA and

$154 \mathrm{CO}_{2}$, underpin the importance of inorganic carbon for biomass synthesis ${ }^{33}$. Different from other

155 Asgard phyla, we did not find type III or type IV ribulose 1,5-bisphosphate carboxylase

156 (RuBisCO) (fig. S10), which might function in the nucleotide salvage pathway ${ }^{11,21,26,27 .}$

157 Most reported Asgard genomes contain genes for Glycerol-1-phosphate dehydrogenase

158 (G1PDH) responsible for ether-bound phospholipids synthesis ${ }^{12,} 33$. A recent study reported a co-

159 existence of enzymes for both ether- (G1PDH) and ester-bound (bacterial/eukarya type, glycerol-

160 3-phosphate dehydrogenase $(\mathrm{G} 3 \mathrm{PDH})$ ) phospholipid synthesis in some Lokiarchaeotal genomes,

161 providing a hint that Asgard archaea might produce chimeric lipids ${ }^{12}$. Intriguingly, Gerdarchaeota

162 MAGs lack the key enzyme G1PDH for archaeal lipid biosynthesis, but contain the

163 bacterial/eukarya-type G3PDH for synthesis of bona fide bacterial lipids (Fig. 2). This finding

164 indicated that Gerdarchaeota might have evolved a bacterial-like membrane predates the

165 eukaryogenesis. Further investigation suggests that the Asgard archaeal G3PDH is synthesized by 166 the $g \operatorname{lpA}$ (responsible for the reverse reaction catalyzed by GpsA) instead of GpsA, which means 
that G3PDH may participate in organic carbon degradation rather than lipid synthesis ${ }^{34}$, 35 , leaving the mechanism of lipid synthesis to be further explored.

\section{Transcriptomic activities of Asgard archaea in different niches of coastal sediments}

Gene expression patterns derived from metatranscriptomic analysis have been used in a number of recent studies to deduce active microbial processes in marine sediments, especially in the deep sea ${ }^{36-38}$. This technique might be constrained by the largely unknown pool size of mRNAs maintained in endospores and dormant cells ${ }^{39}$, although the former seems to be irrelevant for Asgard archaea. Through recruiting 16S rDNA/RNA sequences ( $\mathrm{n}=10,448$, read length >600bp) belonging to Asgard archaea from public databases, we found that all Asgard phyla were transcriptionally expressed and diversely distributed (with 92\% of Asgard OTUs originated from sediment samples, fig. S11 and table S9). Thus, to better uncover their activities, we used 818,479 transcripts (mRNA) belonging to Asgard archaea including Loki-, Thor-, Hel-, Heimdall- and Gerdarchaeota from mangrove sediments to elucidate their ecological roles in coastal sediments.

Organic carbons in coastal sediments are mainly composed of carbohydrates, amino acids, and lipids ${ }^{22,}{ }^{40}$; here, amino acids are the most dominant component of organics in mangrove sediments $^{41}$. Accordingly, we detected high expression levels of genes encoding extracellular peptidases, $\mathrm{ABC}$ transporter, and the enzyme sets for the conversion of amino acids to acetylCoA in both surface and subsurface coastal sediments, implying that Asgard archaea might be essential participants in the degradation of these substrates (Fig. 2, fig. S5, and tables S6, S7 and S10). This feature is supported by the high proportion of peptidases in Asgard archaea MAGs (4.1-6.3\% of the functional genes, fig. S8A). We also detected transcripts for ethanol metabolism, suggesting that ethanol might be another substrate or product. Although glucose accounts for 6- 
$19118 \%$ of the total organic carbon in mangrove sediments ${ }^{41}$, it might be not the first nutritional

choice for Asgard archaea because no transcript for the key enzyme glucokinase was detected.

Due to its higher energetic efficiency compared to fermentative processes ${ }^{43}$, aerobic

respiration contributes to $50 \%$ or more of the total organic matter decomposition in the offshore

marine sediments. The expressed gene set for aerobic respiration in Gerdarchaeota,

Heimdallarchaeota-AAG and Heimdallarchaeota-MHVG, including the key transcript of

cytochrome $c$ oxidase (belonging to Gerdarchaeota) indicates that these Asgard archaea might

participate aerobically in organic matter degradation in surface sediments (Fig. 3). Although co-

existing within the same depth layers, As gard archaea might play phylum specific ecological roles.

For example, unlike Heimdallarchaeota-AAG and Heimdallarchaeota-MHVG, Gerdarchaeota

contain and expressed genes for autotrophy and cellulose degradation, but lack the complete gene

set for glucose degradation (Fig. 3). Like Gerdarchaeota, other Asgard archaea have the potential

to perform anaerobic metabolisms (e.g., acetogenesis) under anoxic conditions (subsurface layers)

42. Notably, Helarchaeota-like mcrA gene transcripts found in unbinned scaffolds (e.g.,

SZ_4_scaffold_203331_2, fig. S12) highlight the involvement of Helarchaeota in alkane

oxidation in coastal sediments, in which ethane and butane might originate from oil-gas seepage

or human activities $s^{43}$, are preferentially used as revealed by molecular modelling and dynamics

studies (fig. S13 and Supplementary Results).

Previous studies suggested that Asgard archaea could account for up to 50\% of the total

210 prokaryotes in some marine sediments ${ }^{44}$; and attribute to $40 \%$ of the total archaeal sequences in

211 coastal sediments ${ }^{44,45}$. The discovery of novel lineages, as well as the discovery of co-occuring

212 diverse lineages in one vertical biosphere in this study, may elevate their relative abundance and

213 ecological roles in natural environments. Therefore, we propose that Asgard archaea might be

214 essential archaeal lineages for organic carbon degradation in coastal sediments, similar to

215 previously proposed roles for Bathyarchaeota ${ }^{45}$ and Thermoprofundales ${ }^{46}$, which also support the 
notion that Asgard archaea are critical participants for organic matter utilization in coastal

217 sediments $44,47$.

\section{Conclusion}

Currently, the research of the diversity and ecology of Asgard archaea is still in its infancy.

221 Contrasting previous studies that Asgard archaeal MAGs covered not more than three phyla ${ }^{3,4,14}$, 222 our study features a wider taxonomic breadth, as we obtained almost all known Asgard phyla in 223 the coastal sediments, and additionally found a new Asgard archaea phylum. Metabolic compartments of organic matter (e.g., peptides and amino acids). Thus, considering their high

227 relative abundance ${ }^{44,45}$ and ubiquitous distribution in the coastal area, we infer that Asgard 228 archaea are important players for organic matter utilization ${ }^{45}, 48$. However, their contribution to 229 the coastal sediment carbon budget remains to be further examined. Overall, the metabolic 230 features, transcript evidence, and their global distribution imply that Asgard archaea are essential 231 players in carbon cycling of coastal sediments.

\section{Materials and Methods}

\section{Sediment sample collection and processing}

Samples for metagenome analysis were collected from the coastal sediment (i.e., mangrove, mudflat and seagrass sediments) of China and Helgoland coastal mud area during the RV HEINCKE cruise HE443 (table S1). They were sampled using custom corers, sealed in plastic bags in duplicates, stored in sampling box with ice bags, and transported to the lab within 4 hours.

The physiochemical parameters of the samples were determined as previously described ${ }^{49}$.

Samples for RNA extraction were preserved in RNALater (Ambion, Life Technologies). For each 
241 sample, $10 \mathrm{~g}$ of sediment each was used for DNA and RNA isolation with the PowerSoil DNA

242 Isolation Kit (MO BIO) and RNA Powersoil ${ }^{\mathrm{TM}}$ Total RNA Isolation Kit (QIAGEN), respectively.

243 The rRNA genes were removed from the total RNA using the Ribo-Zero rRNA removal kit

244 (Illumina, Inc., San Diego, CA, USA) and the remaining mRNA was reverse-transcribed. DNA

245 and cDNA were sequenced using an Illumina HiSeq sequencer (Illumina) with 150-bp paired-end

246 reads at BerryGenomics (Beijing, China). Metatranscriptomic reads were quality-trimmed using

247 Sickle (version 1.33$)^{50}$ with quality score $\geq 25$, and the potential rRNA reads were removed using

248 SortMeRNA (version 2.0) ${ }^{51}$ against both the SILVA 132 database and the default databases (E-

249 value cutoff $\leq 1 \mathrm{e}-5)$

Metagenomic assembly, genome binning and gene annotation

Raw metagenomic DNA reads of the coastal sediments were dereplicated (identical reads)

253 and trimmed using Sickle (version 1.33$)^{50}$ with the option "-q 25". Paired-end Illumina reads for

254 each sample were assembled de novo using IDBA-UD (version 1.1 .1$)^{52}$ with the parameters "-

255 mink 65, -maxk 145, -steps 10". Scaffolds were binned into genomic bins using a combination of

256 MetaBAT $^{20}$ and Das Tool ${ }^{21}$. Briefly, twelve sets of parameters were set for MetaBAT binning,

257 and Das Tool was further applied to obtain an optimized, non-redundant set of bins. To improve

258 the quality of the bins (e.g., scaffold length and bin completeness), each Asgard-related bin was

259 remapped with the short-read mapper BWA and re-assembled using SPAdes (version 3.0.0) ${ }^{53}$ or

260 IDBA-UD (version 1.1.1) ${ }^{52}$, followed by MetaBAT and Das Tool binning. Asgard MAGs with

261 high contamination were further refined with Anvi'o software (version 2.2.2) ${ }^{54}$. The completeness,

262 contamination and strain heterogeneity of the genomic bins were estimated by CheckM (version

263 1.0.7) software ${ }^{55}$. Anvi'o software (version 2.2 .2$)^{54}$ was applied for pan-genome analysis of

264 Asgard MAGs with the option "--min-occurrence 3". 
265 Protein-coding regions were predicted using Prodigal (version 2.6.3) with the "-p meta" option $^{56}$. The KEGG server (BlastKOALA) ${ }^{57}$, eggNOG-mapper ${ }^{58}$, InterProScan tool (V60) ${ }^{59}$, and

267 BLASTp vs. NCBI-nr database searched on December 2017 (E-value cutoff $\leq 1 \mathrm{e}-5$ ) were used to $\mathrm{PSORTb}^{62}($ table S11).

\section{Phylogenetic analyses of Asgard MAGs}

The 16S rRNA gene sequences and a concatenated set of 122 archaeal-specific conserved marker genes ${ }^{63}$ were used for phylogenetic analyses of Asgard archaea. Ribosomal RNA genes in the Asgard MAGs were extracted by Barrnap (version 0.3, http://www.vicbioinformatics.com/software.barrnap.html). An updated 16S rRNA gene sequence dataset from reference papers ${ }^{64,65}$ with genome-based $16 \mathrm{~S}$ rRNA genes were aligned using SINA (version 1.2.11) ${ }^{66}$. The $16 \mathrm{~S}$ rRNA gene sequences maximum-likelihood tree was built with IQTREE (version 1.6.1) ${ }^{67}$ using the GTR+I+G4 mixture model (recommended by the "TESTONLY" model), with option “-bb 1000”. Marker genes for protein tree were identified using hidden Markov models (HMMs) and were aligned separately using hmmalign from HMMER3 ${ }^{68}$ with default parameters. The 122 archaeal marker genes were identified using hidden Markov models. Each protein was individually aligned using hmmalign ${ }^{69}$. The concatenated alignment was trimmed by BMGE with flags "-t AA -m BLOSUM30"70. Then, maximum-likelihood trees were built using IQ-TREE with the best-fit model of "LG+F+R10" followed by extended model selection with FreeRate heterogeneity and 1000 times ultrafast bootstrapping. The final tree was rooted with the DPANN superphylum and Euryarchaeota. 
Potential metabolic pathways were reconstructed based on the predicted annotations and the

291 reference pathways depicted in $\mathrm{KEGG}$ and $\mathrm{MetaCyc}^{71}$. Metatranscriptome data from mangrove

292 and mudflat sediments of Shenzhen Bay (table S1) were analyzed to clarify the transcriptomic

293 activity of Asgard archaea. The abundance of transcripts for each gene was determined by

294 mapping all non-rRNA transcripts to predicted genes using BWA with default setting ${ }^{36,72 .}$

295 Normalized expression was expressed in transcript per million units (TPM), followed by

296 normalization by genome number of each phylum.

\section{ESP identification}

As predicted by prodigal (v2.6.3) with default parameters, genes of Gerdarchaeota were

300 searched against InterPro and eggNOG databases to gain the IPRs and arCOGs. The list of those 301 annotations in Zaremba-Niedzwiedzka et al (2017) ${ }^{3}$ was searched for in the Gerdarchaeota bins.

302 We also manually inspected the IPRs and arCOGs only present in Gerdarchaeota. MAFFT-linsi

303 and trimAl (-gappyout) were used to align and trim the protein sequences. IQ-TREE (version 


\section{References and Notes}

307 1. Spang, A. et al. Complex archaea that bridge the gap between prokaryotes and eukaryotes.

$308 \quad$ Nature 521, 173-179 (2015).

309 2. Seitz, K. W. et al. Genomic reconstruction of a novel, deeply branched sediment archaeal

310 phylum with pathways for acetogenesis and sulfur reduction. ISME J. 10, 1696 (2016).

$3113 . \quad$ Zaremba-Niedzwiedzka, K. et al. Asgard archaea illuminate the origin of eukaryotic

312 cellular complexity. Nature 541, 353-358 (2017).

313 4. Seitz, K. W. et al. Asgard archaea capable of anaerobic hydrocarbon cycling. Nat.

314 Commun. 10, $1822(2019)$.

315 5. Vetriani, C. et al. Population structure and phylogenetic characterization of marine benthic 316 archaea in deep-sea sediments. Appl. Environ. Microbiol. 65, 4375-4384 (1999).

317 6. Inagaki, F. et al. Archaeology of Archaea: geomicrobiological record of Pleistocene

318 thermal events concealed in a deep-sea subseafloor environment. Extremophiles 5, 385-392

319 (2001).

320 7. Takai, K. \& Horikoshi, K. Genetic diversity of archaea in deep-sea hydrothermal vent 321 environments. Genetics 152, 1285-1297 (1999).

322 8. Inagaki, F. et al. Microbial communities associated with geological horizons in coastal 323 subseafloor sediments from the Sea of Okhotsk. Appl. Environ. Microbiol. 69, 7224-7235 (2003).

324 9. Zhou, Z., Liu, Y., Li, M. \& Gu, J. D. Two or three domains: a new view of tree of life in 325 the genomics era. Appl. Microbiol. Biot. 102, 3049-3058 (2018).

326 10. Sousa, F. L. et al. Lokiarchaeon is hydrogen dependent. Nat. Microbiol. 1, 16034 (2016).

327 11. Liu, Y. et al. Comparative genomic inference suggests mixotrophic lifestyle for

328 Thorarchaeota. ISME J. 12, 1021-1031 (2018).

329 12. Manoharan, L. et al. Metagenomes from Coastal Marine Sediments Give Insights into the 330 Ecological Role and Cellular Features of Loki- and Thorarchaeota. mBio 10, e02039-19 (2019). 
331 13. Pushkarev, A. et al. A distinct abundant group of microbial rhodopsins discovered using

332 functional metagenomics. Nature 558, 595-599 (2018).

333 14. Bulzu, P.-A. et al. Casting light on Asgardarchaeota metabolism in a sunlit microoxic 334 niche. Nat. Microbiol. 4, 1129-1137 (2019).

335 15. MacLeod, F. et al. Asgard archaea: Diversity, function, and evolutionary implications in a 336 range of microbiomes. AIMS Microbiol. 5, 48-61 (2019).

337 16. Huang, J.-M., Baker, B. J., Li, J.-T. \& Wang, Y. New microbial lineages capable of 338 carbon fixation and nutrient cycling in deep-sea sediments of the northern South China Sea. Appl. 339 Environ. Microbiol. 85, e00523-19(2019).

340 17. Mcleod, E. et al. A blueprint for blue carbon: toward an improved understanding of the 341 role of vegetated coastal habitats in sequestering CO2. Front. Ecol. Environ. 9, 552-560 (2011).

342 18. Breithaupt, J. L. et al. Organic carbon burial rates in mangrove sediments: Strengthening 343 the global budget. Global Biogeochem. Cy. 26, (2012).

344 19. Kennedy, H. et al. Seagrass sediments as a global carbon sink: Isotopic constraints. Global 345 Biogeochem. Cy. 24, (2010).

346 20. Kang, D. D., Froula, J., Egan, R. \& Wang, Z. MetaBAT, an efficient tool for accurately 347 reconstructing single genomes from complex microbial communities. PeerJ 3, e1165 (2015).

348 21. Sieber, C. M. et al. Recovery of genomes from metagenomes via a dereplication, 349 aggregation and scoring strategy. Nat. Microbiol. 3, 836-843 (2018).

350 22. Miyatake, T., MacGregor, B. J. \& Boschker, H. T. Depth-related differences in organic 351 substrate utilization by major microbial groups in intertidal marine sediment. Appl. Environ. 352 Microbiol. 79, 389-392(2013).

353 23. Silberstein, S., Collins, P. G., Kelleher, D. J. \& Gilmore, R. The essential OST2 gene 354 encodes the 16-kD subunit of the yeast oligosaccharyltransferase, a highly conserved protein 355 expressed in diverse eukaryotic organisms. J. Cell Biol. 131, 371-383 (1995). 
356 24. Marreiros, B. C. et al. Exploring membrane respiratory chains. BBA-Bioenergetics 1857, $357 \quad 1039-1067(2016)$.

358 25. Segerer, A., Neuner, A., Kristjansson, J. K. \& Stetter, K. O. Acidianus infernus gen. nov., 359 sp. nov., and Acidianus brierleyi comb. nov.: facultatively aerobic, extremely acidophilic 360 thermophilic sulfur-metabolizing archaebacteria. Int. J. Syst. Evol. Micr. 36, 559-564 (1986).

361 26. Konishi, Y., Asai, S., Tokushige, M. \& Suzuki, T. Kinetics of the bioleaching of 362 chalcopyrite concentrate by acidophilic thermophile Acidianus brierleyi. Biotechnol. Prog. 15, $363 \quad 681-688(1999)$

364 27. De Rosa, E. et al. [NiFe]-hydrogenase is essential for cyanobacterium Synechocystis sp. 365 PCC 6803 aerobic growth in the dark. Sci. Rep. 5, 12424 (2015).

366 28. Thiel, V. Archaea. Encyclopedia of Geobiology, 64-69 (2011).

367 29. Greening, C. et al. Genomic and metagenomic surveys of hydrogenase distribution 368 indicate $\mathrm{H}_{2}$ is a widely utilised energy source for microbial growth and survival. ISME J. 10, 761$369777(2016)$

370 30. Peters, J. W. et al. [FeFe]- and [NiFe]-hydrogenase diversity, mechanism, and maturation. $371 \quad$ BBA-Mol. Cell Res. 1853, 1350-1369 (2015).

372 31. Hua, Z.-S. et al. Genomic inference of the metabolism and evolution of the archaeal 373 phylum Aigarchaeota. Nat. Commun. 9, 2832 (2018).

374 32. Spang, A. et al. Proposal of the reverse flow model for the origin of the eukaryotic cell 375 based on comparative analyses of Asgard archaeal metabolism. Nat. Microbiol., 1138-1148 376 (2019).

377 33. Yin, X. et al. $\mathrm{CO}_{2}$ conversion to methane and biomass in obligate methylotrophic 378 methanogens in marine sediments. ISME J. 13, 2107-2119 (2019).

379 34. Yokobori, S.-i., Nakajima, Y., Akanuma, S. \& Yamagishi, A. Birth of archaeal cells: 380 molecular phylogenetic analyses of G1P dehydrogenase, G3P dehydrogenases, and glycerol 
381 kinase suggest derived features of archaeal membranes having G1P polar lipids. Archaea 2016, 382 (2016).

383 35. Villanueva, L., Schouten, S. \& Damsté, J. S. S. Phylogenomic analysis of lipid 384 biosynthetic genes of Archaea shed light on the 'lipid divide'. Environ. Microbiol. 19, 54-69 385 (2017).

386 36. Li, M. et al. Genomic and transcriptomic evidence for scavenging of diverse organic 387 compounds by widespread deep-sea archaea. Nat. Commun. 6, 8933 (2015).

388 37. Orsi, W. D., Edgcomb, V. P., Christman, G. D. \& Biddle, J. F. Gene expression in the 389 deep biosphere. Nature 499, 205 (2013).

390 38. Lloyd, K. G. et al. Phylogenetically novel uncultured microbial cells dominate Earth 391 microbiomes. MSystems 3, e00055-18 (2018).

392 39. Bergkessel, M., Basta, D. W. \& Newman, D. K. The physiology of growth arrest: uniting 393 molecular and environmental microbiology. Nat. Rev. Microbiol. 14, 549 (2016).

394 40. Burdige, D. J. Preservation of Organic Matter in Marine Sediments: Controls, 395 Mechanisms, and an Imbalance in Sediment Organic Carbon Budgets? Chem. Rev. 107, 467-485 396 (2007).

397 41. Kristensen, E., Bouillon, S., Dittmar, T. \& Marchand, C. Organic carbon dynamics in 398 mangrove ecosystems: a review. Auqat. Bot. 89, 201-219(2008).

399 42. Sørensen, J., Jørgensen, B. B. \& Revsbech, N. P. A comparison of oxygen, nitrate, and 400 sulfate respiration in coastal marine sediments. Microb. Ecol. 5, 105-115 (1979).

401 43. Zhang, Y. \& Zhai, W.-D. Shallow-ocean methane leakage and degassing to the 402 atmosphere: triggered by offshore oil-gas and methane hydrate explorations. Front. Mar. Sci. 2, 403 (2015).

404 44. Jørgensen, S. L. et al. Quantitative and phylogenetic study of the Deep Sea Archaeal 405 Group in sediments of the Arctic mid-ocean spreading ridge. Front. Microbiol. 4, 299 (2013). 
45. Pan, J. et al. Vertical Distribution of Bathyarchaeotal Communities in Mangrove Wetlands

Suggests Distinct Niche Preference of Bathyarchaeota Subgroup 6. Microb. Ecol. 77, 417-428 (2019).

46. Zhou, Z. et al. Genomic and transcriptomic insights into the ecology and metabolism of benthic archaeal cosmopolitan, Thermoprofundales (MBG-D archaea). ISME J., 1 (2018).

47. Biddle, J. F. et al. Heterotrophic Archaea dominate sedimentary subsurface ecosystems off Peru. Proc. Natl. Acad. Sci. USA 103, 3846-3851 (2006).

48. Zhang, C. J. et al. Prokaryotic Diversity in Mangrove Sediments across Southeastern China Fundamentally Differs from That in Other Biomes. mSystems 4, e00442-19 (2019).

49. Zhou, Z. et al. Stratified bacterial and archaeal community in mangrove and intertidal wetland mudflats revealed by high throughput $16 \mathrm{~S}$ rRNA gene sequencing. Front. Microbiol. 8, (2017).

50. Joshi, N. \& Fass, J. Sickle: A sliding-window, adaptive, quality-based trimming tool for FastQ files (Version 1.33)[Software]. (2011).

51. Kopylova, E., Noé, L. \& Touzet, H. SortMeRNA: fast and accurate filtering of ribosomal RNAs in metatranscriptomic data. Bioinformatics 28, 3211-3217 (2012).

52. Peng, Y., Leung, H. C., Yiu, S. M. \& Chin, F. Y. IDBA-UD: a de novo assembler for single-cell and metagenomic sequencing data with highly uneven depth. Bioinformatics 28, 14201428 (2012).

53. Bankevich, A. et al. SPAdes: a new genome assembly algorithm and its applications to single-cell sequencing. J. Comput. Biol. 19, 455-477 (2012).

54. Delmont, T. O.\& Eren, A. M. Linking pangenomes and metagenomes: the Prochlorococcus metapangenome. PeerJ 6, e4320 (2018).

55. Parks, D. H. et al. CheckM: assessing the quality of microbial genomes recovered from isolates, single cells, and metagenomes. Genome Res. 25, 1043-1055 (2015). 
431 56. Hyatt, D. et al. Prodigal: prokaryotic gene recognition and translation initiation site

432 identification. BMC Bioinformatics 11, 119 (2010).

433 57. Kanehisa, M., Sato, Y. \& Morishima, K. BlastKOALA and GhostKOALA: KEGG tools

434 for functional characterization of genome and metagenome sequences. J. Mol. Biol. 428, 726-731

435 (2016).

436 58. Huerta-Cepas, J. et al. Fast genome-wide functional annotation through orthology

437 assignment by eggNOG-mapper. Mol. Biol. Evol. 34, 2115-2122 (2017).

438 59. Jones, P. et al. InterProScan 5: genome-scale protein function classification.

439 Bioinformatics 30, 1236-1240 (2014).

440 60. Rawlings, N. D., Barrett, A. J. \& Finn, R. Twenty years of the MEROPS database of

441 proteolytic enzymes, their substrates and inhibitors. Nucleic Acids Res. 44, D343-D350 (2015).

442 61. Bagos, P. et al. Prediction of signal peptides in archaea. Protein Eng. Des. Sel. 22, 27-35

443 (2008).

444 62. $\mathrm{Yu}, \mathrm{N}$. Y. et al. PSORTb 3.0: improved protein subcellular localization prediction with 445 refined localization subcategories and predictive capabilities for all prokaryotes. Bioinformatics

446 26, 1608-1615 (2010).

447 63. Vanwonterghem, I. et al. Methylotrophic methanogenesis discovered in the archaeal 448 phylum Verstraetearchaeota. Nat. Microbiol. 1, 16170 (2016).

449 64. Spang, A., Caceres, E. F. \& Ettema, T. J. Genomic exploration of the diversity, ecology, 450 and evolution of the archaeal domain of life. Science 357, eaaf3883 (2017).

451 65. Durbin, A. M. \& Teske, A. Archaea in organic-lean and organic-rich marine subsurface 452 sediments: an environmental gradient reflected in distinct phylogenetic lineages. Front. Microbiol.

$453 \quad 3,168(2012)$.

454 66. Pruesse, E., Peplies, J. \& Glöckner, F. O. SINA: accurate high-throughput multiple 455 sequence alignment of ribosomal RNA genes. Bioinformatics 28, 1823-1829 (2012). 
456 67. Nguyen, L. T., Schmidt, H. A., von Haeseler, A. \& Minh, B. Q. IQ-TREE: a fast and

457 effective stochastic algorithm for estimating maximum-likelihood phylogenies. Mol. Biol. Evol.

$458 \quad 32,268-274(2014)$.

459 68. Mistry, J. et al. Challenges in homology search: HMMER3 and convergent evolution of 460 coiled-coil regions. Nucleic Acids Res. 41, e121 (2013).

461 69. Eddy, S. R. Accelerated Profile HMM Searches. PLoS Comput. Biol. 7, e1002195 (2011).

462 70. Criscuolo, A. \& Gribaldo, S. BMGE (Block Mapping and Gathering with Entropy): a new 463 software for selection of phylogenetic informative regions from multiple sequence alignments.

464 BMC Evol. Biol. 10, 210 (2010).

465 71. Caspi, R. et al. The MetaCyc Database of metabolic pathways and enzymes and the 466 BioCyc collection of Pathway/Genome Databases. Nucleic Acids Res. 36, D623-D631 (2007).

467 72. Li, H. \& Durbin, R. Fast and accurate short read alignment with Burrows-Wheeler 468 transform. Bioinformatics 25, 1754-1760 (2009). 


\section{Acknowledgments}

General: We thank Dr. Nidhi Singh for her suggestions in molecular modeling. We thank the captain, crew and scientists of R/V HEINCKE expeditions HE443

Funding: This research was financed by the National Natural Science Foundation of China (No. 91851105, 31622002, 31970105, 31600093, and 31700430), the Science and Technology Innovation Committee of Shenzhen (Grant No. JCYJ20170818091727570), the Key Project of Department of Education of Guangdong Province (No. 2017KZDXM071), the China Postdoctoral Science Foundation (No. 2018M633111), the DFG (Deutsche Forschungsgemeinschaft) Cluster of Excellence EXC 309 "The Ocean in the Earth System - MARUM - Center for Marine Environmental Sciences" (project ID 49926684) and the University of Bremen.

Author contributions: M.L., M.C. and Y.L. conceived this study. M.C. analyzed the 16S rRNA data, metagenomic data and metatranscriptomic data. Y.L. collected samples and analyzed the metagenomic data. X.Y., M.W.F., T.R.H., R.N., and A.K. provided metagenomic data. Z.Z. provided support for diversity analysis. M.C., Y.C.Y., J.P. and Z.Z. prepared the DNA and cDNA for sequencing. W.L. and X.W. analyzed MCR complex protein structure and simulated the binding substrates. M.C., Y.L., and M.L. wrote, and all authors edited and approved the manuscript.

Competing interests: The authors declare no conflicts of interest. 
Data and materials availability: Archaeal $16 \mathrm{~S}$ rRNA gene sequences were retrieved from NCBI database, SILVA SSU r132 database, and a reference paper as described in and MG-RAST. The newly obtained Asgard MAGs and metatranscriptomic data are available in NCBI database under the project PRJNA495098 and PRJNA360036. 
Figures and Tables
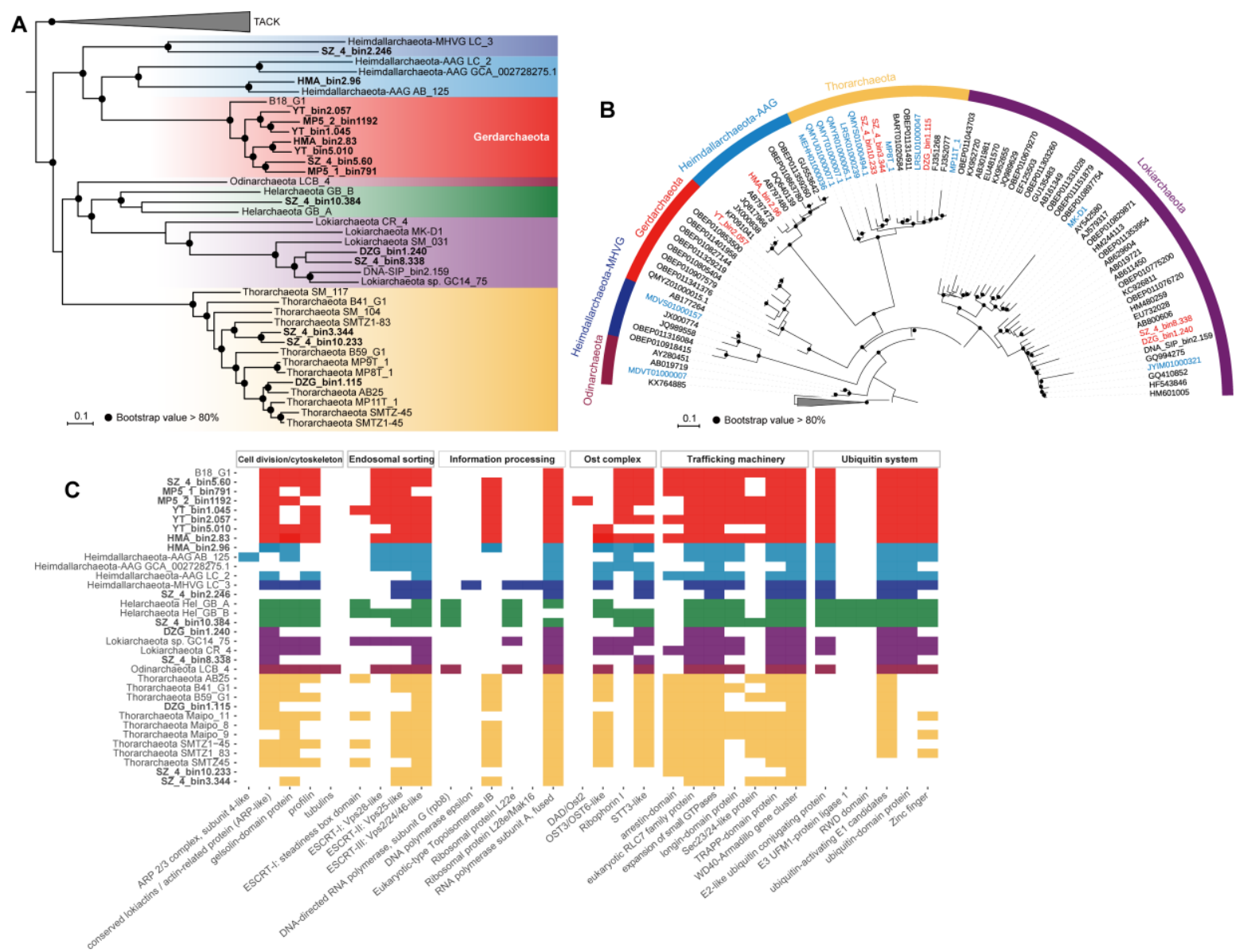

Fig. 1. Phylogenetic positions and ESPs of Asgard archaea reconstructed from coastal

sediments. (A), Maximum likelihood tree of Gerdarchaeota MAGs built on a concatenated

alignment of 122 archaeal marker genes. The tree was inferred with $\mathrm{LG}+\mathrm{F}+\mathrm{R} 10$ mixture mode in

IQ-TREE and rooted with DPANN and Euryarchaeota. Asgard archaea MAGs obtained in this

study are marked in bold face. (B), Phylogenetic position of Asgard archaeal 16S rRNA genes.

511 Red colour represents 16S rRNA genes from newly discovered Asgard MAGs, and blue colour

512 represents sequences from reference ones. The tree was built using the IQ-TREE software with

513 GTR+I+G4 mixture mode and rooted with Crenarchaeota. (C), ESPs identified in Gerdarchaeota

514 and other Asgard archaea. Asgard archaea MAGs obtained in this study are marked bold. Colours

515 indicate phylum-level assignment (see 3A). 


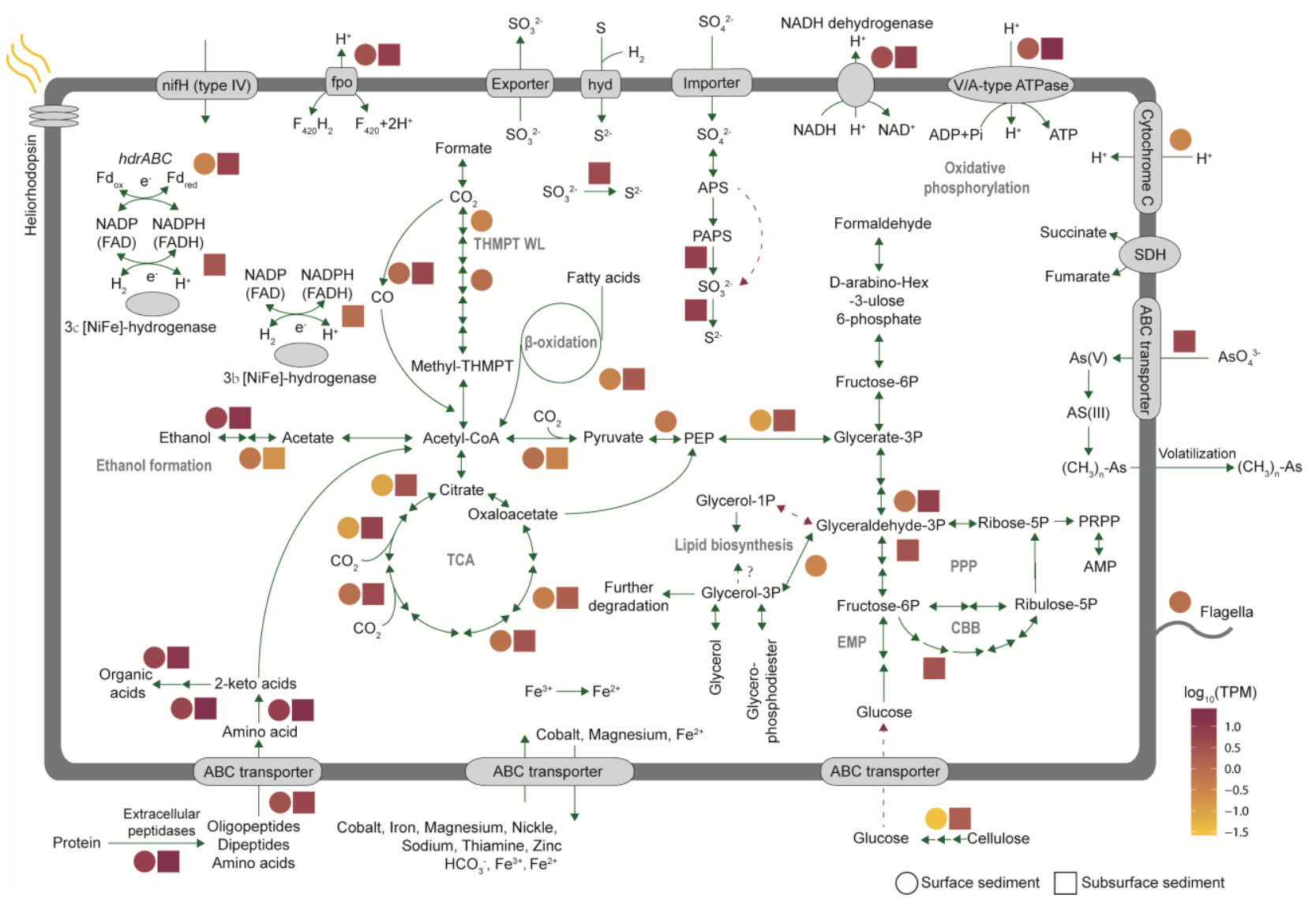

517 Fig. 2. Key potential metabolic pathways of Gerdarchaeota. Solid lines represent pathway

518 steps present in 8 MAGs assigned to Gerdarchaeota. Dashed lines represent pathway steps not

519 identified. Circles represent transcripts from surface sediments and rectangles represents

520 transcripts from subsurface samples. The relative abundance of the transcripts (Transcripts Per

521 Million reads, TPM) for each gene is marked with different colours. Pathway abbreviations: PPP,

522 pentose phosphate pathway (archaea); CBB, Calvin-Benson-Bassham cycle; EMP, Embden-

523 Meyerhof-Parnas pathway, THMPT WL, tetrahydromethanopterin-dependent Wood-Ljungdahl

524 pathway; TCA cycle, tricarboxylic acid cycle. Detailed metabolic information for the MAGs is

525 available in fig. S5, tables S6, and S7. 


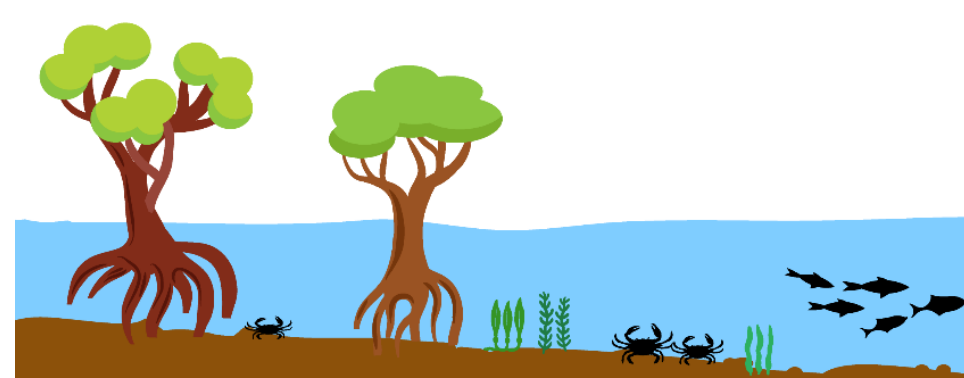

Surface sediment

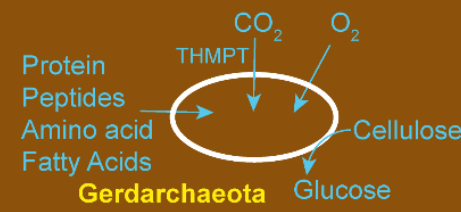

\section{Protein}

Peptides

Amino acid

Fatty Acids

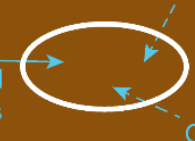

$\mathrm{O}_{2}$

$\mathrm{O}_{2}$

Heimdallarchaeota-MHVG

Protein

Peptides

Amino acid

Fatty Acids

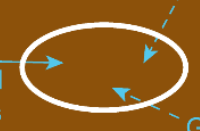

Glucose

Heimdallarchaeota-AAG

Subsurface sediment
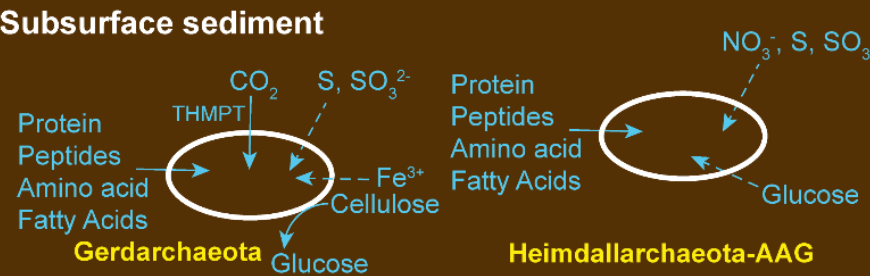

Heimdallarchaeota-AAG

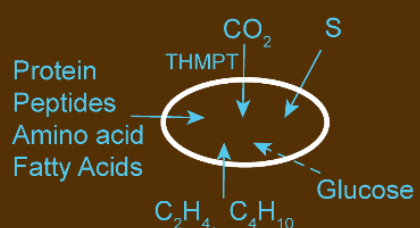

Helarchaeota

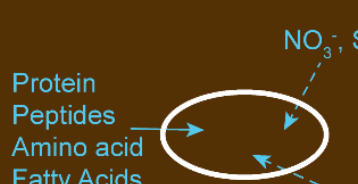

Glucose

Heimdallarchaeota-MHVG

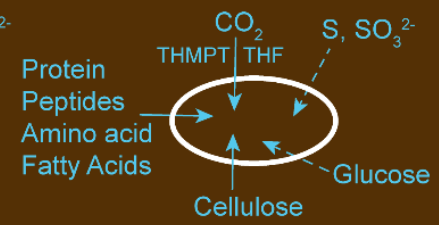

Lokiarchaeota

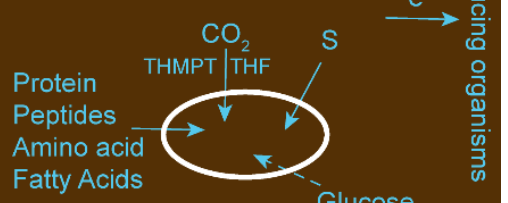

Glucose

Thorarchaeota

Fig. 3. Ecological niches of Asgard archaea in coastal sediments. Dashed lines represent

530 pathways with no transcript for the key genes. Detailed information is available in fig. S5, tables

$531 \quad$ S6, and S7.

533 Supplementary Materials

Supplementary methods and results

figures $\mathrm{S} 1$ to $\mathrm{S} 16$

tables $\mathrm{S} 1$ to $\mathrm{S} 12$ 